\title{
Plastic Surgeon
}

National Cancer Institute

\section{Source}

National Cancer Institute. Plastic Surgeon. NCI Thesaurus. Code C17857.

A surgeon with advanced training in treating disfiguring conditions. Surgery may also include cosmetic surgery. 